\title{
ON REGULAR POINTS OF CONTINUA AND REGULAR CURVES OF AT MOST ORDER $n^{*}$
}

\author{
BY G. T. WHYBURN
}

1. Introduction. In this paper it will be shown, as a consequence of some more general results, that if $n$ is any integer $\neq 2$, the set of all points of order $n$ of any continuum $M$ in a locally compact metric and separable space is punctiform, that is, contains no continuum; and hence there exists no continuum every point of which is of order exactly $n$.

The ordinary notation and terminology of point set theory will be employed. For example, $\bar{X}=X+X^{\prime}$, where $X^{\prime}$ is the set of all limit points of the set $X ; K \cdot H$ means the set of points common to $K$ and $H ; K \subset H$ means that $K$ is a subset of $H$ and $K \supset H$ that $K$ contains $H ; \delta(M)$ denotes the diameter of the set $M ; \rho(X, Y)$ denotes the minimum distance between the sets $X$ and $Y$; if $R$ is an open set, $F(R)$ denotes the boundary of $R$ relative to the whole space, and if $R$ is an open subset of a set $M, F_{m}(R)$ denotes the boundary of $R$ relative to $M$, that is, the set of all those points of $M-R$ which are limit points of $R$. By a continuous curve is meant any connected im kleinen continuum. A neighborhood of a point is an open set containing that point. A point $P$ of a continuum $M$ is called a Menger regular point $\dagger$ of $M$, or simply a regular point of $M$, if for each $\epsilon>0, P$ can be $\epsilon$ separated $\ddagger$ in $M$ by some finite subset of $M$, that is, a finite subset $U$ of $M$ exists such that $M-U=M_{1}+M_{2}$, where $M_{1}$ and $M_{2}$ are mutually separated, $M_{1} \supset P$, and $\delta\left(M_{1}\right)<\epsilon$. If an integer $n$ exists such that, for each $\epsilon>0$, the $\epsilon$-separating set $U$ can be chosen of power $n$, but cannot, (for every $\epsilon$ ), be

* Presented to the Society, Southwestern Section, December 1, 1928.

$\dagger$ See K. Menger, Grundzuge einer Theorie der Kurven, Mathematische Annalen, vol. 95 (1925), pp. 277-306.

$\ddagger$ P. Urysohn, Sur la ramification des lignes Cantoriennes, Comptes Rendus, vol. 175 (1922), p. 481. 
chosen of power $<n, P$ is said to be a point of order $n$ of $M$. A continuum all of whose points are regular points is called a Menger regular curve, or simply a regular curve; and a continuum $M$ such that for some integer $n$, all the points of $M$ are of order $\leqq n$, is called a regular curve of at most order $n$. $^{*}$

Definition. The point $P$ of a continuum $M$ will be said to separate locally a given subset $N$ of $M$ in $M$ if there exists a compact neighborhood $G$ of $P$ such that if $R$ is any neighborhood of $P$ lying in $G$, then $M \cdot \bar{R}-P$ is separated between some two points of $N \cdot R$, that is, $M \cdot \bar{R}-P=M_{1}+M_{2}$, where $M_{1}$ and $M_{2}$ are mutually separated and $M_{1} \cdot N \cdot R \neq 0 \neq M_{2} \cdot N \cdot R$.

The point $P$ of a continuum $M$ is called a local separating point $\dagger$ of $M$ if a compact neighborhood $R$ of $P$ exists such that $M \cdot \bar{R}-P$ is separated between some two points of the component $C$ of $M \cdot \bar{R}$ which contains $P$. Obviously every local separating point of a continuum $M$ locally separates $M$ in $M$. However, the converse is not necessarily true unless $P$ is a regular point of $M$.

2. Theorem 1. If for the closed subset $N$ of a continuum $M$ there exists an integer $n$ such that the set $K$ of points of $N$ which are points of order $\leqq n$ of $M$ and which locally separate $N$ in $M$ is dense in $N$, then the points of $M$ of order $\leqq n / 2+1$ are dense in $N$.

Proof. Let $R$ be any open subset of $N$. By hypothesis $K \cdot R$ contains a point $P_{1}$. There exists a compact open subset $R_{1}$ of $M$ containing $P_{1}$ and such that $N \cdot \bar{R}_{1} \subset R, \delta\left(R_{1}\right)<1$, $F_{m}\left(R_{1}\right)$ is of power $\leqq n$, and $\bar{R}_{1}-P_{1}=R_{11}+R_{12}$, where $R_{11}$ and $R_{12}$ are mutually separated and $R_{11} \cdot N \cdot R_{1} \neq 0 \neq R_{12} \cdot N \cdot R_{1}$. There exists at least one of the sets $R_{11}$ and $R_{12}$, call one such set $D_{1}$, such that $D_{1} \cdot F_{m}\left(R_{1}\right)$ is of power $\leqq n / 2$. Then since $F_{m}\left(D_{1}\right) \subset P+D_{1} \cdot F_{m}\left(R_{1}\right)$, it follows that $F_{m}\left(D_{1}\right)$

* See K. Menger, loc. cit., and K. Menger, Zur allgemeinen Kurventheorie, Fundamenta Mathematica, vol. 10 (1927), pp. 96-115.

† See my paper, Local separating points of continua, presented to the Society October 27, 1928, appearing in Monatshefte für Mathematik und Physik, vol. 36 (1929), No. 2. 
is of power $\leqq n / 2+1$. Clearly $\bar{D}_{1} \cdot N \subset R$ and $\delta\left(D_{1}\right)<1$. Let $N_{1}$ denote the set of points $\bar{D}_{1} \cdot N$. By hypothesis, there exists a point $P_{2}$ in $K \cdot N_{1} \cdot\left[\bar{D}_{1}-F_{m}\left(D_{1}\right)\right]$. There exists an open subset $R_{2}$ of $M$ containing $P_{2}$ and such that $\bar{R}_{2} \subset \bar{D}_{1}$ $-F_{m}\left(D_{1}\right), \delta\left(R_{2}\right)<1 / 2, F_{m}\left(R_{2}\right)$ is of power $\leqq n$, and $\bar{R}_{2}-P$ $=R_{21}+R_{22}$, two mutually separated sets with $R_{21} \cdot N \cdot R_{2}$ $\neq 0 \neq R_{22} \cdot N \cdot R_{2}$. For at least one of the sets $R_{21}$ and $R_{22}$, call one such set $D_{2}$, it is true that $D_{2} \cdot F_{m}\left(R_{2}\right)$ is of power $\leqq n / 2$. Then, just as in the case of $D_{1}$, it follows that $F_{m}\left(D_{2}\right)$ is of power $\leqq n / 2+1$ and that $\bar{D}_{2} \subset \bar{D}_{1}-F_{m}\left(D_{1}\right)$ and $\delta\left(D_{2}\right)<1 / 2$. Let $N_{2}=\bar{D}_{2} \cdot N$. Then $N_{2} \subset N_{1}$, and there exists a point $P_{3}$ in $K \cdot \bar{N}_{2} \cdot\left[\bar{D}_{2}-F_{m}\left(D_{2}\right)\right]$. Just as above it follows that there exists a subset $D_{3}$ of $D_{2}$ containing $P_{3}$ and such that $D_{3} \cdot N_{2} \neq 0, \delta\left(D_{3}\right)<1 / 3, \bar{D}_{3} \subset \bar{D}_{2}-F_{m}\left(D_{2}\right)$, and $F_{m}\left(D_{3}\right)$ is of power $\leqq n / 2+1$. Let this process be continued indefinitely, giving a sequence $D_{1}, D_{2}, D_{3}, \cdots$, of subsets of $M$ such that, for each $i, \bar{D}_{i} \subset \bar{D}_{i-1}-F_{m}\left(D_{i-1}\right), \delta\left(D_{i}\right)<1 / i$, $0 \neq D_{i} \cdot N \subset R$, and $F_{m}\left(D_{i}\right)$ is of power $\leqq n / 2+1$. There exists a point $P$ which belongs to every set $D_{i}$. Then clearly $P$ is a point of order $\leqq n / 2+1$ of $M$. And since $P$ is a limit point of $N$ and $N$ is closed, then $P$ belongs to $N$ and hence to $R$. Thus every open subset of $N$ contains a point of order $\leqq n / 2+1$ of $M$, and our theorem is proved.

COROLLARY 1a. If for a continuum $M$ there exists an integer $n$ such that the local separating points of $M$ of order $\leqq n$ are dense in $M$, then the points of order $\leqq n / 2+1$ are dense in $M$.

THEOREM 2. If $M$ is a Menger regular curve, and if for some integer $n$, all the local separating points of $M$ are of order $\leqq n$, then points of $M$ of order $\leqq n / 2+1$ are dense in $M$.

By a theorem of the author, ${ }^{*}$ the local separating points in a regular curve $M$ are dense in $M$. Then since every local separating point of $M$ locally separates $M$ in $M$, it follows by hypothesis and Theorem 1 that the points of $M$ of order $\leqq n / 2+1$ are dense in $M$.

* See my paper, Concerning points of continuous curves defined by certain im kleinen properties, offered to Mathematische Annalen. 
CoRollary 2a. If for some integer $n$, every point of a continuum $M$ is of order $\leqq n$, that is, if $M$ is a regular curve of at most order $n$, then the points of $M$ of order $\leqq n / 2+1$ are dense in $M$.

COROLlaRy 2b. There does not exist an integer $n \neq 2$ and a continuum $M$ every point of which is of order exactly $n$.

COROLlaRY 2c. The simple closed curve is the only continuum of at most finite order every point of which is of the same order.

THEOREM 3. If Nis any connected set of regular points of a continuum $M$, the set $K$ of all those points of $N$ which are local separating points of $M$ and locally separate $N$ in $M$ is dense in $N$.

Proof. Let $R$ be any open subset of $N$, which we will suppose is $\neq N$, and let $P$ be a point of $R$. Since $P$ is a regular point of $M$, there exists a compact open subset $D$ of $M$ containing $P$ and such that $F_{m}(D)$ is finite and $\bar{D} \cdot N \subset R$. Since $N$ is connected and $D \cdot N \neq 0 \neq N \cdot(M-\bar{D})$, it follows that $F_{m}(D)$ contains a point $X$ of $N$ which is a limit both of $N \cdot D$ and of $N \cdot(M-\bar{D})$. And since $N$ is connected im kleinen, it follows that there exist compact connected subsets $A$ and $B$ of $N$ such that $A \cdot B=X, A-X \subset D$, and $B-X \subset M-\bar{D}$. Let $G$ be a compact neighborhood of $X$ such that $\bar{G} \cdot\left[F_{m}(D)-X\right]=0$. Then if $V$ is any neighborhood of $X$ lying in $G, M \cdot \bar{V}-X=\bar{V} \cdot D+\bar{V} \cdot(M-\bar{D})$; and since $D$ and $M-\bar{D}$ are mutually separated, it follows that $\bar{V} \cdot D$ and $\bar{V} \cdot(M-\bar{D})$ are mutually separated. Clearly these two sets contain points of $A$ and $B$ respectively. Therefore $X$ is a local separating point of $M$ which locally separates $N$ in $M$, and hence it belongs to $K$. Thus, since $X \subset R$, it follows that $K$ is dense in $N$.

THEOREM 4. If $M$ is any continuum and $n$ is any integer $>2$, the set $K^{n}$ of all points of $M$ of order $n$ is punctiform, that is, contains no continuum.

Proof. Suppose, on the contrary, that for some integer $n>2, K^{n}$ contains a continuum $N$. Now by Theorem 3 , the set $K$ of points of $N$ which locally separate $N$ in $M$ is dense in $N$. Then, since every point of $K$ is a point of order $n$ of $M$, it 
follows by Theorem 1 that $N$ contains at least one point of order $\leqq n / 2+1$ of $M$. But since $n>2$, the greatest integer which is less than or equal to $n / 2+1$ is less than $n$. Hence $N$ contains a point of order $<n$ of $M$, contrary to supposition.

Note. Menger (loc. cit.) has shown that the set $K^{1}$ of all points of order 1 (end points) of any continuum $M$ is null dimensional, that is, each of its points can, for each $\epsilon>0$, be $\epsilon$-separated in $K^{1}$ by a null set. Hence, using Theorem 4, if $n \neq 2$, the set $K^{n}$ of points of order $n$ of any continuum is punctiform. It would be interesting to determine whether or not, for $n \neq 2$, the set $K^{n}$ is always null dimensional as well as punctiform. I will remark that the set $K^{w}$ of points of order $w$ (that is, regular points which are of no finite order) of a continuum, or even of a regular curve, is not necessarily punctiform, as can be shown by the curve obtained by making some simple additions to the Sierpinski regular curve.*

THEOREM 5. In any regular curve $M$ of at most order $n$, the set $H$ of points of order $>n / 2+1$ is null dimensional.

Proof. The set $H$ must be punctiform. For suppose it contains a continuum $N$. Then since every point of $N$ is a regular point of $M$ of order $\leqq n$, by Theorem 3 the set $K$ of points of $N$ which locally separate $N$ in $M$ is dense in $N$. Hence, by Theorem $1, N$ contains a point of order $\leqq n / 2+1$, contrary to supposition. Thus $H$ is punctiform. And since by a theorem of Menger, $\nmid H$ is an $F_{\sigma}$ (that is, the sum of a countable number of closed sets), it follows by a theorem of Mazurkiewicz $\ddagger$ that $H$ is homeomorphic with some linear set. Therefore, since $H$ is punctiform, it is $\S$ null dimensional.

We note here the fact that while the set $H$ in Theorem 5 is null dimensional, it is not necessarily countable; for an

\footnotetext{
* See W. Sierpinski, Comptes Rendus, vol. 160 (1915), p. 302.

$\dagger$ Grundzïge einer Theorie der Kurven, loc. cit., Theorem 4. 1913.

$\ddagger$ S. Mazurkiewicz, Bulletin de l'Académie des Sciences de Cracovie,

$\S$ See W. Sierpinski, Sur les ensembles connexes et non connexes, Fundamenta Mathematicae, vol. 2 (1921), p. 89.
} 
example is easily constructed of a regular curve of at most order 3 in which the points of order 3 form a perfect set.

3. Regular Curves of at Most Order 3. Knaster and Kuratowski have shown* that the Sierpinski regular curve, which is a curve of at most order 4 , contains a connected set which contains no perfect subset. In this section I shall show that no curve of at most order 3 can contain any such set, and indeed, that every connected subset of a continuous curve $M$, every maximal cyclic curve of which is a regular curve of at most order 3 , contains a perfect set of local separating points of $M$. The continuous curve $M$ is cyclicly connected $\dagger$ if every two of its points lie together on some simple closed curve in $M$. The cyclicly connected continuous curve $C$ is said to be a maximal cyclic curve of a continuous curve $M$ if $C$ is a subset of $M$ and is not a proper subset of any cyclicly connected continuous curve which is a subset of $M$.

LEMMA 6a. In a regular curve of at most order 3, no two maximal cyclic curves have a common point.

Lemma $6 \mathrm{a}$ follows at once from the facts that no two maximal cyclic curves of a continuous curve can have more than one point in common and that every point of a cyclicly connected curve $C$ is of order not greater than 2 of $C$.

THEOREM 6. If every maximal cyclic curve of a continuous curve' $M$ is a regular curve of at most order 3 , then every connected subset $H$ of $M$ contains a perfect set of local separating points of $M$.

Proof. If every point of $H$ is either a cut point or an end point of $M$, then $\ddagger H$ is arcwise connected and hence contains

* A connected and connected im kleinen point set which contains no perfect subset, this Bulletin, vol. 33 (1927), pp. 106-109.

† See my paper, Cyclicly connected continuous curves, Proceedings of the National Academy of Sciences, vol. 13 (1927), pp. 31-38; for the extension of the results in this paper to a more general space than the plane, see an abstract by W. L. Ayres in this Bulletin, vol. 33 (1927), p. 661.

¥ See my paper, Concerning the structure of a continuous curve, American Journal of Mathematics, vol. 50 (1928), pp. 167-194, Theorem 8; see also an abstract by C. M. Cleveland in this Bulletin, vol. 32 (1926), p. 420. 
an arc every point of which is a cut point of $M$. If not, then $H$ contains at least two points of some maximal cyclic curve $C$ of $M$. Let $W=H \cdot C$. Then* $W$ is connected and contains more than one point. Since, by hypothesis, every point of $W$ is a point of order $\leqq 3$ of $C$, it follows by Theorem 3 that $W$ contains a point $P$ which locally separates $W$ in $C$. Then since $P$ obviously is a point of order $\geqq 2$ of $W$, it follows that a connected open subset $R$ of $C$ exists containing $P$ and such that (1) $F_{c}(R)$ is of power $\leqq 3,(2) \bar{R} \cdot W$ is connected, and (3) $F_{c}(R)$ contains at least two points $A$ and $B$ of $W$ which are separated in $\bar{R}$ by the point $P$. Now since $\bar{R} \subset C, \bar{R}$ is a regular curve of at most order 3 . Hence, by Lemma 6a, no two maximal cyclic curves of $\bar{R}$ have a point in common. And since $A$ and $B$ do not lie in the same maximal cyclic curve of $\bar{R}$, it follows by a theorem of the author $\dagger$ that $A$ and $B$ are separated in $\bar{R}$ by each point of a perfect point set $K$. Now $K \subset W \cdot \bar{R}$, for $W \cdot \bar{R}$ is connected and contains both $A$ and $B$. Clearly every point of $K$ is a local separating point of $M$. Thus $H$ contains a perfect set $K$ of local separating points of $M$, and our theorem is proved.

It would be interesting to determine whether or not, in a regular curve of at most order 3 , every connected subset is arcwise connected, and whether every punctiform subset is null dimensional.

The foregoing results yield immediately the following additional theorem on regular curves of at most order 3 .

THEOREM 7. In a regular curve $M$ of at most order 3 the boundary with respect to $M$ of every connected open subset of $M$ is totally disconnected.

The University of Texas

* G. T. Whyburn, loc. cit., Theorem 30.

† See my paper, Concerning the cyclic elements of continuous curves, presented to the Society September 4, 1928. 